\title{
FRACTALES: AYUDA DIAGNÓSTICA PARA CÉLULAS PRENEOPLÁSICAS Y CANCERÍGENAS DEL EPITELIO ESCAMOSO CERVICAL CONFIRMACIÓN DE APLICABILIDAD CLÍNICA
}

\author{
JAVIER RODRÍGUEZ ${ }^{*}$, SIGNED PRIETO ${ }^{1}$, HÉCTOR POSSO ${ }^{2}$, \\ RICARDO CIFUENTES ${ }^{2}$, CATALINA CORREA ${ }^{1}$, YOLANDA SORACIPA ${ }^{3}$, \\ FREDY LÓPEZ ${ }^{1}$, LAURA MÉNDEZ ${ }^{1}$, HEBERT JAMES BERNAL ${ }^{1}$, ALEJANDRO SALAMANCA ${ }^{1}$ \\ ${ }^{1}$ Investigadores Grupo Insight. Línea de Profundización e Internado Especial Física y Matemáticas \\ Aplicadas a la Medicina, Universidad Militar Nueva Granada. \\ Centro de Investigaciones Clínica del Country. Bogotá, Colombia.
}

${ }^{2}$ Investigador Grupo Insight. Docente Facultad Medicina. Universidad Militar Nueva Granada. Bogotá, Colombia. Centro de Investigaciones Clínica del Country. Bogotá, Colombia.grupoinsight2025@yahoo.es

${ }^{3}$ Lic. Fisica. Docente Facultad Ciencias Básicas. Universidad Nacional Abierta y a distancia. Investigadora Grupo Insight. Bogotá, Colombia.

Recibido: Noviembre 15 de 2014

Aceptado: Octubre 28 de 2015

\section{Resumen}

Antecedentes: Se desarrolló un método diagnóstico fractal para evaluar células del cuello uterino utilizando el concepto de Armonía Matemática Intrínseca (AMI) y variabilidad celular, el cual diferencia matemáticamente células normales de células L-SIL y H-SIL, haciendo innecesario el diagnóstico de células ASCUS.

Objetivo: confirmar la capacidad diagnóstica de la metodología desarrollada mediante un estudio ciego de comparación con el Gold Standard.

Método: se tomaron fotografías digitales de 50 preparaciones citológicas de mujeres entre 20 y 55 años: 5 con diagnóstico de citología normal y 45 con diferentes grados de lesión hasta carcinoma, incluyendo 5 ASCUS. Se calculó la dimensión fractal de tres objetos matemáticos: núcleo, citoplasma y totalidad, a partir de la superposición de cinco rejillas. Además, se evaluó su dimensión fractal mediante el concepto de AMI y variabilidad celular. Los resultados obtenidos se compararon con el diagnóstico citopatológico convencional determinando su sensibilidad, especificidad y coeficiente kappa.

Resultados: La sensibilidad y especificidad fue del 100\%, y el coeficiente Kappa de 1.

Conclusiones: Los resultados en una población diferente a la inicial son una evidencia de la capacidad de esta metodología para diagnosticar objetiva y cuantitativamente células normales, L-SIL y H-SIL, así como aclarar el diagnóstico de las células ASCUS con base en la dimensión fractal y el concepto de AMI y variabilidad.

Palabras Clave: Cáncer, Cuello uterino, Fractal, Citología, Diagnóstico. 


\title{
FRACTALS: DIAGNOSTIC HELP FOR PRENEOPLASTIC AND CANCERIGINAL CELLS OF CERVICAL SCALPAL EPITHELIUM CONFIRMATION OF CLINICAL APPLICABILITY
}

\begin{abstract}
Introduction: A new fractal diagnostic method was applied for assessing cervix cells using the Intrinsic Mathematical Harmony (IMH) concept and cellular variability, which mathematically differentiates normal, L-SIL and H-SIL cells making unnecessary the diagnostic of ASCUS cells.

Objective: To confirm the diagnostic capability of developed methodology by a blind study of comparison with Gold Standard.

Methods: Digital pictures were taken of 50 cells from cytology of 20 to 55 year-old women; 5 with normal diagnosis and 45 with several lesion degrees until carcinoma, including 5 with ASCUS diagnosis. Fractal dimension of three mathematical objects was calculated by superposing five grids: nucleus, cytoplasm and totality. Moreover, their fractal dimension was assessed through the Intrinsic Mathematical Harmony concept and cellular variability. The obtained diagnosis was compared with conventional cytopathologic diagnosis determining sensibility, specificity and Kappa coefficient.
\end{abstract}

Results: $100 \%$ Sensibility and specificity, and a Kappa Coefficient of 1 were obtained.

Conclusions: The results, in a population different to the initial one, are an evidence of the ability of this methodology for objectively and quantitatively diagnosing the normal, L-SIL and H-SIL cells, as well as for clarifying the ASCUS cells diagnosis, based on fractal dimension and IMH concept and variability.

Keywords: Cancer, Cervix, Fractal, Cytology, Diagnosis. 


\section{FRACTALES: AJUDA DIAGNÓSTICA PARA CÉLULAS PRENEOPLÁSTICAS E CANCERIGINAS DO EPITELIO DO SCALPEL CERVICAL CONFIRMAÇÃO DA APLICABILIDADE CLÍNICA}

\section{Resumo}

Introdução: Um novo método de diagnóstico fractal foi aplicado para avaliar as células do colo do útero usando conceito intrínseco harmonia matemática (IMH) e variabilidade celular, que normalmente diferencia matematicamente, LSIL e HSIL células que fazem o diagnóstico de células desnecessários ASCUS.

Objetivo: Para confirmar a capacidade de diagnóstico da metodologia desenvolvida por um estudo cego de comparação com Gold Standard.

Métodos: Foram tiradas fotos digitais de 50 células de citologia de mulheres com 20 a 55 anos de idade; 5 com diagnóstico normal e 45 com vários graus de lesão até carcinoma, incluindo 5 com diagnóstico de ASCUS. A dimensão fractal de três objetos matemáticos foi calculada através da superposição de cinco grades: núcleo, citoplasma e totalidade. Além disso, sua dimensão fractal foi avaliada através do conceito de harmonia matemática Intrínseca e da variabilidade celular. O diagnóstico obtido foi comparado com o diagnóstico citopatológico convencional, determinando a sensibilidade, especificidade e coeficiente Kappa.

Resultados: Foram obtidos 100\% de sensibilidade e especificidade e um Coeficiente Kappa de 1.

Conclusões: Os resultados, em uma população diferente da inicial, são uma evidência da habilidade desta metodologia para o diagnóstico objetivo e quantitativo das células normais, L-SIL e H-SIL, bem como para esclarecer o diagnóstico das células ASCUS, Baseado na dimensão fractal e no conceito e variabilidade de IMH.

Palavras-chave: Câncer, Cérvix, Fractal, Citologia, Diagnóstico. 


\section{Introducción}

En Colombia el cáncer de cuello uterino ocupa el primer lugar en cáncer presentado en mujeres, y es uno de los países que presenta las tasas más altas de incidencia de esta patología. Según estudios recientes el cáncer de cuello uterino ocupa el segundo lugar de incidencia en el mundo entre los tumores malignos de la mujer, causando 231.000 muertes por año (1). Además, es el cuarto tipo de cáncer más frecuente en mujeres en el continente americano, y es el segundo más frecuente en mujeres entre 15 y 44 años (2). El 70.7\% de los cánceres cervicales invasivos se atribuyen al papiloma-virus de diferentes tipos, infección que portan cerca del $14,5 \%$ de las mujeres; sin embargo, el papiloma-virus es considerado un factor necesario, pero no suficiente para el desarrollo de cáncer de cuello uterino.

En la actualidad la citología cérvico-uterina es la técnica de tamizaje más empleada, ya que esta evalúa las características morfológicas de las células de cuello uterino y determina la presencia de anormalidades que puedan ser sospechosas de malignidad. Dado que se considera que los carcinomas cervicales son el estado final de una serie de alteraciones epiteliales progresivamente atípicas que evolucionan en ausencia de sintomas, resulta de vital importancia a nivel preventivo detectar las lesiones precursoras que aparecen en el cérvix y que pueden evolucionar hacia cáncer invasivo, siendo el Sistema Bethesda la clasificación más empleada internacionalmente (3), que destaca la elaboración de un sistema binario para catalogar las anormalidades celulares preneoplásicas en el extendido citológico, denominándolas Lesiones Intraepiteliales Escamosas de Alto o Bajo grado (H-SIL y L-SIL, respectivamente).

Sin embargo, el Sistema Bethesda no ha sido adoptado globalmente en todos los países (3), dado que este se fundamenta en observaciones cualitativas y subjetivas de la morfología celular, generando indeterminaciones diagnósticas en muchos casos, catalogadas como ASCUS. Las pruebas de índole morfológica tal como se evalúan actualmente son de carácter subjetivo y por lo tanto la correlación entre las observaciones hechas en citología, colposcopia y biopsia no siempre es adecuada; al respecto algunos especialistas afirman que la notable discrepancia entre el aspecto morfológico de un tumor y su comportamiento biológico genera ocasionalmente predicciones erradas (4). Todo esto redunda en que la evaluación realizada no provee la información necesaria para predecir la futura evolución de una neoplasia.

La irregularidad del cuerpo humano es una característica propia de éste. Sin embargo, tradicionalmente se ha medido con métricas euclidianas, mediante la aproximación de sus formas a líneas, áreas y volúmenes regulares. Como respuesta a esta imposibilidad de hacer medidas confiables a esta clase de objetos, se desarrolla la geometría fractal la cual permite caracterizar adecuadamente la forma irregular del cuerpo humano (5). A partir de la geometría fractal se obtiene la dimensión fractal que es una medida numérica que representa el grado de irregularidad de un objeto (6). Existen diferentes metodologías para calcular la dimensión fractal que dependen de si el objeto a medir es un objeto abstracto, salvaje o estadístico. Específicamente, los objetos salvajes que se caracterizan por superposición de sus partes se miden con el método de Box-Counting (7-9).

La aplicación de la dimensión fractal dentro del campo de la medicina ha sido de gran importancia en las áreas clínica y experimental, en las que se han caracterizado objetos como la estructura neuronal y pulmonar, los intestinos y los vasos sanguíneos de diferentes estructuras, entre otros (10). En cuanto a estudios morfométricos realizados en cáncer, se ha aplicado la geometría fractal proporcionando una medida más objetiva de aplicación clínica (11-16). Específicamente en el área de la oncología se ha logrado caracterizar estructuras cancerígenas $(11,12,17)$, imágenes mamográficas (18), e identificar la aparición de cáncer primario en casos de metástasis en huesos (5).

En estudios realizados siguiendo líneas similares de trabajo, Rodríguez et al realizaron una investigación descriptiva de las características fractales de las células escamosas de cuello uterino, que habían sido clasificadas por el sistema Bethesda como normales, ASCUS y L-SIL, encontrando una caracterización del grado de irregularidad de dichas células y hallando una organización fractal de su estructura (19). Posteriormente, se desarrolló un método de evaluación diagnóstica fractal que permitió hallar diferencias matemáticas entre las células clasificadas como normales y como L-SIL, y encontrando que las células clasificadas como ASCUS o tenían valores matemáticos asociados a normalidad o a L-SIL (20). Esta metodología representa la primera evaluación cuantitativa de valor preventivo para la salud pública en el área de prevención de lesiones preneoplásicas y neoplásicas de cuello uterino. 
Con base en los dos trabajos previos, se generó una nueva metodología en la que se cuantifican las relaciones fractales de las células preneoplásicas y neoplásicas de las células escamosas de cuello uterino, las cuales fueron evaluadas a partir de la dimensión fractal calculada para tres objetos celulares definidos como son: el núcleo, el citoplasma sin núcleo y la totalidad celular. Así mismo, se midió la variabilidad y Armonía Matemática Intrínseca de estas células. Adicionalmente, se hallaron todos los prototipos celulares representantes de cada estadio entre normalidad y carcinoma obteniéndose una generalización aplicable a la práctica clínica. En este estudio se encontraron 18 prototipos de normalidad, mientras que se encontraron 44 prototipos de anormalidad, dentro de los que se incluyen 8 de carcinoma (21).

El desarrollo de métodos cuantitativos para la evaluación de la forma de la célula escamosa del epitelio del cuello uterino ofrece la ventaja de ser objetiva y reproducible, superando las dificultades de variabilidad interobservador de los métodos de evaluación que dependen de la experiencia del especialista. Estos métodos desarrollados, además, evidenciaron la no justificación, desde un punto de vista matemático, de la existencia del diagnóstico de ASCUS, dado que los tipos celulares estudiados se comportaron o como normales o como lesiones de bajo grado $(20,21)$.

El propósito del presente estudio es confirmar la aplicabilidad clínica de la metodología diagnóstica desarrollada (21) con base en las dimensiones fractales y los conceptos de AMI y variabilidad, para la evaluación de células de cuello uterino con diferentes grados de alteración celular hasta carcinoma, comparando el diagnóstico matemático y el diagnóstico convencional mediante medidas de sensibilidad, especificidad y coeficiente kappa.

\section{Metodología}

\section{Definiciones}

Dimensión fractal: medida usada para cuantificar el grado de irregularidad de un objeto. El método más utilizado para la cuantificación de la irregularidad de objetos que presentan superposición de sus partes es el método de Box-Counting.

Dimensión Fractal de Box-Counting:

$D=\frac{\log \mathrm{N}\left(2^{-(K+1)}\right)-\log \mathrm{N}\left(2^{-K}\right)}{\log 2^{k+1}-\log 2^{t}}=\log _{2} \frac{N\left(2^{-(i+1)}\right)}{N\left(2^{-k}\right)}$

Ecuación 1
En esta expresión $\boldsymbol{N}$ corresponde al número de cuadros que contiene el contorno del objeto, $K$ corresponde al grado de partición de la cuadrícula, y $\boldsymbol{D}$ representa la dimensión fractal.

AMI celular y Variabilidad: Corresponde al Grado de similitud o diferencia entre las dimensiones fractales de dos de los objetos definidos, en este caso se compararán el citoplasma sin núcleo y núcleo $(\mathrm{C}-\mathrm{N})$, el núcleo y la totalidad (N-T) y citoplasma sin núcleo y totalidad (C-T). Se obtiene hallando la diferencia entre la primera cifra decimal de las dimensiones fractales de los dos objetos que se van a comparar.

Prototipo celular fractal: corresponde a una combinación simultánea de relaciones numéricas entre las dimensiones fractales de los objetos estudiados: núcleo $(\mathrm{N})$, citoplasma sin núcleo $(\mathrm{C})$ y totalidad $(\mathrm{T})$ evaluadas con la AMI y Variabilidad.

\section{Procedimiento}

Se seleccionaron 50 imágenes de células provenientes de citologías cérvico-vaginales de mujeres entre los 20 y 55 años, de las cuales 5 tienen un diagnóstico citológico dentro de límites normales, 5 fueron diagnosticadas como ASCUS, 20 con L-SIL y 20 con H-SIL. Estas imágenes fueron obtenidas de la Liga Contra el Cáncer, Seccional Bogotá. Se observaron a un aumento de $100 \mathrm{X}$ a través de microscopio, posteriormente se fotografiaron con una cámara digital para finalmente transferirlas a través de interfase en serie al computador. Las fotografías adquirieron dimensiones de $1200 \mathrm{x}$ 1600 píxeles, y se observaron al $50 \%$ en el computador para su análisis.

Se definieron tres objetos matemáticos a medir, basado en estudios previos (21): núcleo (N), citoplasma sin núcleo $(\mathrm{C})$ y totalidad $(\mathrm{T})$ de cada célula, calculando su dimensión fractal con el método de Box-Counting a partir de cinco rejillas compuestas por cuadros de 2, 4, 8, 16 y $32 \mathrm{~mm}$ de lado.

Sobre las imágenes se calculó la dimensión fractal con el método simplificado de Box-Counting para los tres objetos matemáticos definidos, empleando un software en lenguaje $\mathrm{C}++$ desarrollado previamente. Posteriormente se establecieron sus medidas de AMI celular y Variabilidad para las tres comparaciones obtenidas: $\mathrm{C}$ y N, N y $\mathrm{T}$ y C y $\mathrm{T}$. 
Partiendo de los prototipos de normalidad y anormalidad obtenidas previamente (21), y que determinan su estado de acuerdo con sus medidas de AMI celular y Variabilidad, se determinó su diagnóstico histológico de acuerdo con los parámetros previamente establecidos (21).

Esta investigación se encuentra enmarcada dentro de los principios éticos de la Declaración de Helsinki de la Asociación Médica Mundial, y de acuerdo a la normatividad Colombiana en investigación especificada en el artículo 11 de la resolución 008430 de 1993, del Ministerio de salud, se clasifica como investigación sin riesgo dado que se realizan cálculos físicos sobre resultados de exámenes no invasivos de la práctica clínica que han sido prescritos médicamente, protegiendo la privacidad, integridad y anonimato de las participantes.

\section{Análisis Estadístico}

Se contrastó el resultado matemático obtenido para cada caso, con el resultado diagnosticado por el médico experto según la metodología tradicional, que se asumió como Gold-Standard, comparando este resultado con la metodología matemática calculando la especificidad y la sensibilidad. Las células ASCUS no fueron incluidas debido a que su diagnóstico histológico desde los parámetros convencionales es incierto; sin embargo, se observó su comportamiento matemático con el fin de establecer cuantitativamente su cercanía a los diferentes estados.

Dichas medidas se realizaron a través de una clasificación binaria donde los verdaderos positivos (VP) corresponden el número de células diagnósticas histológicamente dentro de los límites de anormalidad, incluyendo L-SIL y H-SIL, y que se encuentran dentro de los valores matemáticos correspondientes al mismo diagnóstico, los falsos positivos (FP) corresponden al número de muestras que matemáticamente se comportan como estudios dentro de la anormalidad y cuyo diagnóstico histológico es normal, los falsos negativos (FN) corresponden al número de muestras cuyos valores matemáticos son normales pero que histológicamente se corresponden con muestras anormales y los verdaderos negativos (VN) corresponden al número de exámenes diagnosticados histológicamente como normales y cuyos valores matemáticos también se relacionan con normalidad.

Con el objetivo de evaluar la concordancia entre los valores físico matemático y el diagnóstico histológico convencional se calculó el coeficiente Kappa a través de la siguiente fórmula:

$$
K=\frac{C o-C a}{T o-C a}
$$

Donde:

Co: número de concordancias observadas, es decir, número de células con el mismo diagnóstico de acuerdo con la nueva metodología propuesta y con el Gold Standard.

To: totalidad de observaciones, es decir, la totalidad de casos normales y con lesión preneoplásica o neoplásica.

Ca: Concordancias atribuibles al azar, que se calculan de acuerdo con la siguiente fórmula:

\section{$\mathrm{Ca}=\left[\left(f_{1} x C_{1}\right) / \mathrm{To}\right]+\left[\left(f_{2} x C_{2}\right) / \mathrm{To}\right]$}

Donde $\mathrm{f}_{1}$ es el número de células que presentan valores matemáticos dentro de los límites de normalidad, $\mathrm{C}_{1}$ es el número de células diagnósticas histológicamente dentro de la normalidad, $\mathrm{f}_{2}$ es el número de células que presentan valores matemáticos asociados a lesión preneoplásica o neoplásica, $\mathrm{C}_{2}$ es el número células diagnósticas histológicamente con lesión preneoplásica $\mathrm{O}$ neoplásica y To es el número total de casos normales y con lesión preneoplásica o neoplásica.

\section{Resultados}

Las dimensiones fractales del citoplasma de las células normales medidas estuvieron entre 0,1805 y 1,9958; para el núcleo se encontró que las dimensiones fractales estuvieron entre 0,2462 y 1,986 y para la totalidad se obtuvieron valores entre 0,1968 y 1,9805. Los valores de variabilidad y AMI de la comparación entre Citoplasma y Núcleo se hallaron entre 0 y 5 , al igual que entre Núcleo y Totalidad, mientras que para la comparación entre Citoplasma y Totalidad se obtuvieron valores de 0 o 1 (Tabla 1).

Se encontró que las 10 muestras diagnosticadas como normales desde los parámetros convencionales fueron diagnosticadas como normales con base en la metodología físico-matemática, al presentar valores de AMI y variabilidad de $\mathrm{C}$ y $\mathrm{N}$, y de $\mathrm{N}$ y $\mathrm{T}$ entre 3 y 5 en todos los casos, así como valores entre 0 y 1 en la comparación $\mathrm{C}$ y T. Las 20 L-SIL y las $20 \mathrm{H}$-SIL también presentaron el 
mismo diagnóstico matemático e histológico; las primeras presentaron valores entre 0 y 2 para las tres comparaciones en todos los casos, mientras que las segundas presentaron valores de 0 para las tres comparaciones en todos los casos. De este modo, el análisis estadístico dio como resultado una sensibilidad y una especificidad del $100 \%$, y un coeficiente Kappa de 1.
Se encontró adicionalmente que de las cinco células ASCUS evaluadas, una de ellas presentó valores correspondientes a normalidad, tres a L-SIL y una a H-SIL, confirmando que este estadío no presenta características matemáticas que permitan diferenciarla de los otros, y que por el contrario es posible aclarar su diagnóstico mediante este procedimiento, estableciendo si sus medidas matemáticas corresponden a uno u otro estadío (Tabla 1).

Tabla 1. Medidas de dimensión fractal variabilidad fractal y AMI celular de 25 de las muestras evaluadas, incluyendo 4 normales, 5 ASCUS, 7 L-SIL y 9 H-SIL. C: Citoplasma; N: Núcleo; T: Totalidad. Dx: Diagnóstico matemático, que es el mismo diagnóstico convencional en todos los casos a excepción de las células ASCUS, que corresponden a las filas sombreadas, evidenciando que este tipo de células pueden presentar comportamientos matemáticos análogos a normalidad, L-SIL o H-SIL.

\begin{tabular}{|c|c|c|c|c|c|c|c|}
\hline \multirow{2}{*}{ No. } & \multirow{2}{*}{ Dx. } & \multicolumn{3}{|c|}{ DIMENSION FRACTAL } & \multicolumn{3}{|c|}{$\begin{array}{l}\text { VARIABILIDAD FRACTAL } \\
\text { Y AMI CELULAR }\end{array}$} \\
\hline & & $\mathrm{C}$ & $\mathbf{N}$ & $\mathrm{T}$ & C Y N & N Y T & C Y T \\
\hline 1 & H-SIL & 1,5809 & 1,5328 & 1,5379 & 0 & 0 & 0 \\
\hline 2 & H-SIL & 1,6891 & 1,6469 & 1,6596 & 0 & 0 & 0 \\
\hline 3 & H-SIL & 0,9382 & 0,9986 & 0,9521 & 0 & 0 & 0 \\
\hline 4 & H-SIL & 0,7396 & 0,7819 & 0,7168 & 0 & 0 & 0 \\
\hline 5 & H-SIL & 1,6162 & 1,6784 & 1,6309 & 0 & 0 & 0 \\
\hline 6 & H-SIL & 1,5347 & 1,5169 & 1,5831 & 0 & 0 & 0 \\
\hline 7 & H-SIL & 0,2903 & 0,2718 & 0,212 & 0 & 0 & 0 \\
\hline 8 & H-SIL & 0,2002 & 0,2462 & 0,2626 & 0 & 0 & 0 \\
\hline 9 & $\mathrm{H}-\mathrm{SIL}$ & 1,9561 & 1,986 & 1,9457 & 0 & 0 & 0 \\
\hline 10 & H-SIL & 1,153 & 1,1821 & 1,1741 & 0 & 0 & 0 \\
\hline 11 & L-SIL & 0,8489 & 0,6414 & 0,7837 & 2 & 1 & 1 \\
\hline 12 & L-SIL & 1,6535 & 1,5471 & 1,6384 & 1 & 1 & 0 \\
\hline 13 & L-SIL & 1,8618 & 1,6366 & 1,7702 & 2 & 1 & 1 \\
\hline 14 & L-SIL & 1,4479 & 1,5486 & 1,4491 & 1 & 1 & 0 \\
\hline 15 & L-SIL & 0,2488 & 0,4649 & 0,3693 & 2 & 1 & 1 \\
\hline 16 & L-SIL & 0,6945 & 0,6819 & 0,7923 & 0 & 1 & 1 \\
\hline 17 & L-SIL & 0,4104 & 0,6441 & 0,5746 & 2 & 1 & 1 \\
\hline 18 & L-SIL & 1,5053 & 1,6684 & 1,4647 & 1 & 2 & 1 \\
\hline 19 & L-SIL & 0,1805 & 0,3499 & 0,1968 & 2 & 2 & 0 \\
\hline 20 & L-SIL & 1,7576 & 1,5131 & 1,7463 & 2 & 2 & 0 \\
\hline 21 & Normal & 1,6204 & 1,9378 & 1,6716 & 3 & 3 & 0 \\
\hline 22 & Normal & 1,3808 & 1,6855 & 1,3571 & 3 & 3 & 0 \\
\hline 23 & Normal & 1,9958 & 1,4836 & 1,9805 & 5 & 5 & 0 \\
\hline 24 & Normal & 1,4949 & 1,9211 & 1,5202 & 5 & 4 & 1 \\
\hline 25 & Normal & 1,1258 & 1,532 & 1,1783 & 4 & 4 & 0 \\
\hline
\end{tabular}




\section{Discusión}

Este es el primer trabajo en el que se realiza una confirmación de la capacidad diagnóstica de la metodología previamente desarrollada por Rodríguez y col., (21) a partir de la geometría fractal y el concepto de AMI y variabilidad celular. Se estableció una comparación entre el diagnóstico convencional y el físico-matemático, con el propósito de evidenciar el grado de certeza que tiene la metodología para caracterizar los grados de alteración que van desde la normalidad hasta carcinoma, evidenciando que mediante este procedimiento es posible diagnosticar matemáticamente células normales, L-SIL y H-SIL con una sensibilidad y especificidad del $100 \%$ y un coeficiente kappa de 1 respecto al diagnóstico obtenido con base en el Sistema Bethesda, tomado como Gold Standard. Los resultados obtenidos confirman los hallazgos previamente establecidos por Rodríguez y col., (21) en los cuales se demuestra que existe una relación entre la disminución de los valores de la variabilidad y AMI celular en las relaciones $\mathrm{C}-\mathrm{N}$ y N-T, y el aumento de la gravedad de las alteraciones preneoplásicas.

Actualmente, la clasificación de Bethesda es uno de los métodos más ampliamente empleados para la interpretación diagnóstica de las imágenes citopatológicas de las células de cuello uterino ya que, aunque está basada en parámetros cualitativos proporciona una terminología definida y uniforme (22). Diferentes estudios se han realizado para determinar la sensibilidad y especificidad de la prueba diagnóstica citológica, encontrándose una sensibilidad que varía entre 30 y $80 \%$ y una especificidad que varía entre 86 y $100 \%$. Adicionalmente, los estudios indican que es menos específica para lesiones de alto grado que para lesiones de bajo grado (23). Otros autores, en el marco de revisiones sistemáticas han reportado una especificidad de $98 \%$ con una sensibilidad del $51 \%$ (24), con una tasa de falsos negativos de alrededor del 35,5\% (25). Por el contrario, la presente metodología supera la baja sensibilidad reportada en estos estudios debido a que se encuentra fundamentada en medidas geométricas, que por su carácter cuantitativo garantizan la objetividad y reproducibilidad de la interpretación diagnóstica.

Investigaciones basadas también en geometría fractal, han permitido en otros ámbitos de la oncología establecer caracterizaciones útiles y facilitar la comprensión del fenómeno neoplásico. Diferentes estudios se han enfocado hacia la descripción morfológica de distintos tipos de cáncer $(11,12)$, para la identificación del si- tio primario de cáncer en metástasis ósea (26) y en imágenes diagnósticas utilizadas para tamizaje como la mamografía (18). También se ha aplicado al estudio de diferentes fenómenos, tales como la morfología cardiovascular, permitiendo la diferenciación de arterias coronarias normales de las reestenosadas (27), metodología que fue generalizada posteriormente hallando el número total de posibles prototipos arteriales en proceso de reestenosis (28). También, se han desarrollado métodos aplicables a la evaluación de ecocardiografías pediátricas (29), y de la ramificación coronaria de adultos (30).

Otro tipo de teorías físicas y matemáticas se han aplicado a diferentes campos de la medicina aportando soluciones a diferentes problemas y permitiendo el desarrollo de métodos predictivos, como por ejemplo, en el área de la biología molecular aplicando teoría de probabilidad y entropía $(31,32)$, en infectología logrando predicciones del recuento de CD4 aplicando teoría de conjuntos (33), en fisiología cardiaca aplicando probabilidad y entropía (34), o una ley caótica exponencial $(35,36)$ y para la predicción de epidemias aplicando probabilidad y la relación S/k de la entropía (37).

Este trabajo se encuentra enmarcado dentro de una concepción fundamentalmente acausal, que sustenta la abstracción de cualquier correlación de causa-consecuencia que convencionalmente es sostenida en los estudios con metodologías estadísticas lineales que relacionan estadísticamente los fenómenos estudiados con diversas variables como el género, la edad, las comorbilidades y los factores de riesgo, entre otros. Visto este estudio desde una perspectiva física y matemática, no es posible observar en este fenómeno una evolución continua y única en el tiempo, dado que no existe una única trayectoria posible. Para su estudio sólo se cuenta con observaciones discretas, denominadas por Prigogine "ventanas temporales" (38), que permiten su descripción matemática desde una perspectiva acausal. Esta perspectiva acausal, también es compartida en otros contextos científicos que se han establecido a partir del desarrollo de la física moderna, tales como la mecánica estadística $(39,40)$, la teoría del caos $(41,42)$ y la mecánica cuántica $(43)$.

\section{Financiación}

Este trabajo se realizó con el apoyo financiero del Fondo de Investigaciones de la Universidad Militar Nueva 
Granada. Facultad de Medicina. Proyecto MED-1344.

Es importante agradecer al Dr. Fernando Cantor, Vicerrector de Investigaciones, a la Dra. Martha Bahamón, Vicerrectora Académica, al Dr. Jorge Luque, Decano de la Facultad de Medicina y al Teniente Coronel Médico Luis Antonio Castro Gómez, Director del Centro de Investigaciones de la Facultad de Medicina por su confianza y colaboración con nuestros trabajos de investigación. Así mismo, se agradece al Ingeniero Mario Castro, y al Dr. Henry Acuña, por su permanente apoyo a nuestros trabajos.

Queremos agradecer al Centro de Investigaciones de la Clínica del Country, a los Doctores Tito Tulio Roa, Director de Educación Médica, Jorge Ospina, Director Médico, Alfonso Correa, Director del Centro de Investigaciones, y a las Doctoras Adriana Lizbeth, epidemióloga y Silvia Ortiz, Enfermera Jefe del Centro de Investigaciones por su apoyo a nuestras investigaciones.

Agradecemos a la Doctoras del Laboratorio de Patología de la Liga Contra el Cáncer, Martha Vega, citotecnóloga, y Constanza Gómez, Jefe de laboratorio, por proporcionar, para este trabajo, las muestras necesarias.

\section{Conflicto de Intereses}

Los autores declaran no tener de manera directa o indirecta, algún tipo de conflicto de intereses financieros, académicos o laborales que puedan poner en peligro la validez de este estudio.

\section{Referencias}

1. González J, González E, Biete A, Casanova Ll, Mestres J. Cáncer de cérvix. En: González J, González E, Biete A, Casanova Ll, Mestres J. Ginecología Oncológica. $2^{a}$ ed. Barcelona: Masson; 2000.

2. WHO/ICO Information Centre on HPV and Cervical Cancer (HPV Information Centre). Human Papillomavirus and Related Cancers in Americas 2010. Disponible en: www. who.int/hpvcentre

3. Lacruz C. Nomenclatura de las lesiones cervicales (de Papanicolau a Bethesda 2001). Rev Esp Patol. 2003; 36(1):5-10.

4. DeVita VT, Hellman S, Rosenberg S. Principios y práctica de oncología. $5 \mathrm{a}$ ed. Bogotá: Editorial Medica Panamericana, ARAN ediciones S.A.;1997.

5. Vasiljevic J, Reljin B, Sopta J, Mijucic V, Tulic G, Reljin I. Application of multifractal analysis on microscopic images in the classification of metastatic bone disease. Biomed
Microdevices. 2012; 14:541-548.

6. Mandelbrot B. Introducción. En: Mandelbrot B. Los Objetos Fractales. Barcelona. Tusquets Eds. S.A.; 2000. p.13-26.

7. Peitgen, J, Jürgens, H, Saupe, D. Limits and self similarity. En: Chaos and Fractals: New Frontiers of Science. New York: Springer-Verlag. 1992. p.135-182.

8. Peitgen, J, Jürgens, H, Saupe, D. Length area and dimension. Measuring complexity and scalling properties. En: Chaos and Fractals: New Frontiers of Science. New York: Springer-Verlag. 1992. p.183-228.

9. Mandelbrot, B. The Fractal Geometry of Nature. San Francisco: Freeman Ed.; 1972; 341-348.

10. West JW. Fractal physiology and chaos in medicine. Singapore: World Scientific; 1990.

11. Luzi P, Bianciardi G, Miracco C, Desanti MM, Del Vecchio MT, Alia L, et al. Fractal analysis in human pathology. Ann NY Acad Sci. 1999; 879:255-57.

12. Baish H, Jain R. Fractals and Cancer. Cancer research. 2000; 60: 3683- 3688.

13. Pohlman S, Powell K, Obuchowski NA. Quantitative classification of breast tumors in digitized mammograms. Med Phys. 1996; 23:1337-45.

14. Lefebure F, Benali H. A fractal approach to the segmentation of microcalcifications in digital mammograms. Med Phys. 1995; 22:381-3890.

15. Landini G, Rippin JW. Fractal dimensions of epithelial-connective tissue interfaces in premalignant and malignant ephitelial lesions of the floor of mouth. Anal Quant Cytol Histol. 1993; 15:144-49.

16. Gazit Y, Baish JW, Safabaksh N. Fractal characteristics of tumor vascular architecture during tumor growth and regression. Microcirculation. 1997; 4:395-402.

17. Sankar D, Thomas T. A New Fast Fractal Modeling Approach for the Detection of Microcalcifications in Mammograms. Journal of Digital Imaging. 2010; 23(5):538- 546.

18. Stępień R, Stępień P. Analysis of Contours of Tumor Masses in Mammograms by Higuchi's Fractal Dimension. Biocyb Biomed Eng. 2010; 30(4): 49-56.

19. Rodríguez J, Prieto S, Ortiz L, Wiesner C, Díaz M, Correa C. Descripción matemática con dimensiones fractales de células normales y con anormalidades citológicas de cuello uterino. Revista Ciencias de la Salud. 2006; 4(2):58-63.

20. Rodríguez J. Nuevo método de ayuda diagnóstica con Geometría fractal para células preneoplásicas del epitelio escamoso cervical. Revista UDCA Actualidad \& Divulgación Científica. 2011;14(1):15-2

21. Rodríguez J, Prieto S, Correa C, Posso H, Bernal P, Vitery $\mathrm{S}$, et al. Generalización fractal de células preneoplásicas y cancerígenas del epitelio escamoso cervical de aplicación clínica. Rev Med. 2010;18(2):173-181. 
22. Dim CC. Towards improving cervical cancer screening in Nigeria: A review of the basics of cervical neoplasm and cytology. Niger J ClinPract. 2012; 15:247-52.

23. Stoler MH, Schiffman M. Atypical Squamous Cells of Undetermined Significance-Low-grade Squamous Intraepithelial Lesion Triage Study (ALTS) Group. Interobserver reproducibility of cervical cytologic and histologic interpretations: realistic estimates for the ASCUS-LSIL Triage Study. JAMA. 2001; 285:1500-1505.

24. Nanda K, McCrory DC, Myers ER, Bastian LA, Hasselblad $\mathrm{V}$, Hickcy JD, et al. Accuracy of the Papanicolaou test in screening for and follow-up of cervical cytologic abnormalities: a systematic review. Ann Intern Med. 2000; 132: 810-819.

25. Spence AR, Goggin P, Franco EL. Process of care failures in invasive cervical cancer: systematic review and metaanalysis. Prev Med. 2007; 45:93-106.

26. Vasiljevic J, Reljin B, Sopta J, Mijucic V, Tulic G, Reljin I. Application of multifractal analysis on microscopic images in the classification of metastatic bone disease. Biomed Microdevices. 2012; 14:541-548.

27. Rodríguez J, Marino M, Avilan N, Echeverri D. Medidas fractales de arterias coronarias, un modelo experimental en reestenosis armonía matemática intrínseca de la estructura arterial. Rev Col Cardiología. 2002; 10: 65-72.

28. Rodríguez J, Prieto S, Correa C, Bernal P, Puerta G, Vitery $\mathrm{S}$, et al. Theoretical generalization of normal and sick coronary arteries with fractal dimensions and the arterial intrinsic mathematical harmony. BMC Medical Physics. 2010; 10-11.

29. Rodríguez J, Prieto S, Ortiz L, Ronderos M, Correa C. Diagnóstico matemático de ecocardiografías pediátricas con medidas de dimensión fractal evaluadas con armonía matemática intrínseca. Rev Colomb Cardiol. 2010; 17: 79-86.

30. Rodríguez J, Prieto S, Correa C, Bernal P, Tapia D, Álvarez L, et al. Diagnóstico fractal de disfunción cardiaca severa. Dinámica fractal de la ramificación coronaria izquierda. Rev Colomb Cardiol. 2012; 19(5):225-232.

31. Rodríguez J, Berna P, Prieto P, Correa C, Álvarez L, Pinilla L, Tovar, et al. Predicción de unión de péptidos de Plasmodiumfalciparum al HLA clase II. Probabilidad, combinatoria y entropía aplicadas a las proteínas MSP-5 y MSP-6. Archi- vos de alergia e inmunología clínica. 2013; 44(1): 7-14.

32. Rodríguez J. Teoría de unión al HLA clase II teorías de probabilidad combinatoria y entropía aplicadas a secuencias peptídicas. Inmunología. 2008; 27(4):151-166.

33. Rodríguez J, Prieto S, Correa C, Pérez C, Mora J, Bravo $\mathrm{J}$, et al. Predictions of CD4 lymphocytes' count in HIV patients from complete blood count. BMC Medical Physics 2013; 13:3.

34. Rodríguez J, Prieto S, Domínguez D, Melo M, Mendoza F, Correa $\mathrm{C}$, et al. Mathematical-physical prediction of cardiac dynamics using the proportional entropy of dynamic systems. J. Med. Med. Sci. 2013; 4(9):370-381.

35. Rodríguez J, Correa C, Melo M, Domínguez D, Prieto S, Cardona DM, et al. Chaotic cardiac law: Developing predictions of clinical application. J. Med. Med. Sci. 2013;4(2): 79-84.

36. Rodríguez J, Narváez R, Prieto S, Correa C, Bernal P, Aguirre $G$, et al. The mathematical law of chaotic dynamics applied to cardiac arrhythmias. J Med Med Sci. 2013; 4 (7): 291-300.

37. Rodríguez J. Método para la predicción de la dinámica temporal de la malaria en los municipios de Colombia. Rev Panam Salud Pública 2010; 27(3):211-8.

38. Fernández, A. Introducción. Orden y Caos. Barcelona: Labor Prensa Cientifica S.A.; 1990. p. 4-8.

39. Feynman, RP, Leighton, RB, Sands, M. Leyes de la Termodinámica. En: Feynman, RP, Leighton, RB, Sands, M. Física. Wilmington: Addison-Wesley Iberoamericana, S. A. 1964. Vol. 1. p 44.1-44.19

40. Tolman, R. Principles of statistical mechanics. Primeraedición. New York: Dover Publications. 1979.

41. Devaney, R. A first course in chaotic dynamical systems theory and experiments. Reading Mass.: Addison- Wesley 1992.

42. Crutchfield J, Farmer D, Packard N, Shaw R. Caos. En: Crutchfield J, Farmer D, Packard N, Shaw R. Orden y Caos. Barcelona: Labor Prensa Cientifica S.A.; 1990. p. 78-90.

43. Feynman, R, Leighton, R, Sands M. Comportamiento cuántico. Vol. 1, cap.37. En: Feynman R, Leighton R, Sands M. Física. Wilmington: Addison-Wesley Iberoamericana, S. A.; 1964. 